\title{
Physical Activity and Emotional Status of College Students and Working Sector on 1 Month Lockdown
}

\author{
Isha Garg ${ }^{1}$, Shahiduz Zafar ${ }^{2}$, Sajjad Alam ${ }^{3}$, Rituraj Verma ${ }^{3}$, \\ Yamini Sharma ${ }^{4}$, Megha Yadav ${ }^{4}$, Anchal Bisht ${ }^{1}$ \\ ${ }^{1}$ BPT Student, Dept of Physiotherapy, Galgotias University, Greater Noida, India, ${ }^{2}$ Professor, Department of \\ Physiotherapy, Galgotias University, Greater Noida, India, ${ }^{3}$ Associate Professor, Dept of Physiotherapy, \\ Galgotias University, Greater Noida, India, ${ }^{4}$ Assistant Professor, Dept of Physiotherapy, Galgotias University, \\ Greater Noida, India
}

\begin{abstract}
Introduction: Due to COVID-19 pandemic outbreak, a lot of countries are under lockdown for more than 30 days. The study is about the physical activity and emotional status of college students and the working sector for 1 month lockdown.
\end{abstract}

Aim: To check the physical and emotional status of college students and in the working sector, while the response is either positive or negative towards the health concern.

Method: A web survey was used which is based on IPAQ and PERS scales by 150 candidates.

Result: IPAQ show the physical activity perform by the 150 candidates where the maximum candidate was of low activity and PERS shows the positive and negative reactivity of the candidates where the negative reactivity was high than positive reactivity.

Conclusion: The low physical activity stimulates the negative reactivity.

Keywords: COVID-19, IPAQ AND PERS, Physical and Emotional Status.

\section{Introduction:}

As the COVID-19 has a globally pandemic effect on whole globalization. Due to which the people have to be quarantined from more than 30 days for the welfare of each person who can be treated from COVID-19. This lockdown will help to locate the people who are being symptomatic with COVID-19 and are not yet located or reported. Therefore the lockdown has an effect on one's human body in many ways and the two factors which are more affected are physical and emotional factors .Human existence is possible if there is correlation between

\footnotetext{
Corresponding Author:

Isha Garg

BPT Student, Dept of Physiotherapy,Galgotias

University, Greater Noida, India

e-mail: isha.garg@galgotiasuniversity.edu.in
}

the neurobiological and integration of movement for the proper function of the human body and to balance this equation movement and mental health plays an important role.Due to restrictions for COVID-19 there is a great impact on the physical activity which is not properly followed by college students and the workplace. BECAUSE these two sectors are the most active groups of the society which perform vigorous to moderate types activity . The sudden lockdown may associate with sudden physical inactivity which further associate with insulin resistance in muscle tissue and even there is an decreased glucose utilization in muscle which further cause the muscle atrophy ${ }^{4}$. Therefore the sudden cessation of physical inactivity can also cause the decrease in the rate of blood flow back to the heart and reduced the coronary blood pressure ${ }^{5}$.

The World Health Organization (WHO) has provided some information to follow over the physical activity during this lockdown which is essential as well 
as beneficial for each of us for the proper functioning of the body and even for the balance of adequate health.

The guidelines are such that a individual should engage at least

(a) $150 \mathrm{~min} /$ week on moderate intensity physical activity

(b) $75 \mathrm{~min} /$ week on vigorous/high intensity physical activity

(c) or, the combination of both moderate-vigorous/high physical activity ${ }^{2}$.

DUE to prolonged time of lockdown the physical inactivity also affects the bone and joint health due to inadequate stimulation in muscles ${ }^{7}$. The doctors say that with such a long time period of lockdown will not only affect the physical activity but also overall and general health in mainly patients like diabetes and hypertension will also have an affect on mental status? Physical inactivity also affects the mental health which can be examined by unpleasant emotions such as anger, fear, sadness, happiness, frustration, irritation etc. Psychological disorders like post traumatic stress, post traumatic depression and post traumatic confusion among people was followed by the author's like Brooks ${ }^{3}$. The impact on emotional inactivity is much depress by the anxiety among people in days of lockdown ${ }^{6}$. Reports of China, about the psychological health of people over there cause fear-anxiety, increased domestic violence and babies being abandoned ${ }^{8}$. The effect of lockdown on mental stability is they have fear of spreading virus, about the family welfare, phobias and anxiety are prioritizing the current situation ${ }^{8}$. The lockdown is not providing the platform to meet loved ones with each other which is also the major cause of depression ${ }^{7}$. The news that are occurring can cause more anxiety and stress on people during

lockdown ${ }^{7}$.. The alcohol consumption in this lockdown is increased due to stress and certain emotions that emerging within individuals ${ }^{7}$. The lockdown period is such an emotional phase for each one due to which there is an intense fear among the people which causes them panic attacks on future delaying, the health status of one wellbeing [7]. As the habit of working either physically or mentally during not lockdown or simple routine lifestyle caught into the trap of COVID-19 in bad terms. The impact of covid-19 for one month lockdown creates an impact on physical health, mental health as well as life satisfaction which is supported by Dr.
ZHANG ${ }^{9}$. Work helps people in their wellbeing,in their state of mind through the daily routine, through some hopes and purpose in life ${ }^{9}$. Reports claim that during this lockdown if the sedentary lifestyle of youth is increased more than demand will be a major cause of depression and anxiety ${ }^{10}$. The impact of covid-19 is directly affecting the financial income of employees ${ }^{8}$. The sector are allowedto work through telecommunication ${ }^{2}$.To work on these two sector with two factors .the scales are used for further reports. The scale which is used is IPAQ scale international physical activity questionnaire .the scale is used to measure the activity related that is perform within population ${ }^{11}$. The scale consist of two types of activity

\section{(a) Vigorous type activity \\ (b) Moderate type activity \\ (c) No activity}

That a person does in their routine for the last 7 days. The other scale which is used to measure the emotional events during this lockdown is by PERS scale (Perth Emotional Reactivity Scale). The scale has several measurements which depend on one's response that are based on activation, intensity, and duration questionnaire that are proposed in two ways positive and negative. The purpose of the topic is to find out the physical activity and emotional status on the college student as well as on the working sector during this one month lockdown ${ }^{6}$.

\section{Method}

Description: During this pandemic outbreak of covid-19. The college and all the working

The sector is locked down under the government orders. The proverb is "Prevention is better than cure" during this locked down how the physical and emotional inactivity is analyzed between these two sectors where the data is collected on the basis of web surveys.

Study Population: The population which is taken in this web survey was the population of college students and the working sector. The survey is based on physical inactivity and emotional inactivity on the population during a one month lockdown. There are150 individuals which based their answers through the questionnaire survey.

Survey Instrument: The web survey was based on the multiple choice questions, it took approximately 10 minutes to complete the survey. The two different scales 
are used for two different factors, questions explored the following:-

(a) The knowledge or concern of physical inactivity among individuals

(b) The stability of emotional status among the two sectors

(c) The impact of lockdown is positive or negative in context of physical and emotional health.

The impact of physical inactivity is checked by the IPAQ scale 'International physical activity questionnaire' where the questionnaire is based on 2 types of activity, vigorous activity and moderate activity in the last 7 days which is done between the age of 15-69 ${ }^{12}$. The impact of emotional inactivity is checked by the PERS scale " Perth emotional reactivity scale' is the questionnaire to measure the reactions of emotions at emotional events . The scale has several measurements which depend on one's response that are based on activation, intensity, and duration questionnaire that are proposed in two ways positive and negative ${ }^{13}$.

Statistical Analysis: The collection of data is based on a web survey questionnaire which is formed in Google forms.

The survey consists of questionnaires which are of physical activity and emotional status.

Physical Activity: The physical activity data is based on the components that is determined by the IPAQ scale. The components are:

(a) No activity.

(b) Mild/low activity.

(c) Moderate activity.

(d) Vigorous/high activity.

The components give the detailed general analysis of hours and minutes that is collected by 150 respondents according to activity they performed during 1 month lockdown.

(a) No activity: the no activity includes (sitting for long hours). The data shows $44.2 \%$ responders sits for within 6 hours while $2.4 \%$ sits above 18 hours .

(b) Mild activity: The mild activity includes the activity of walking, the data and analysis represents the days, hours and minutes by the sectors(working sector and college students). (c) Moderate Activity: Moderate activities refer to activities that where the oxygen uptake is moderate higher than the normal. . The examples of moderate activities are carrying little loads, bicycling at a pace, or play doubles tennis which does not include walking. Therefore the data that is collected is based on the days/week, hours an minutes/day

(d) Vigorous/High activity: Vigorous physical activities refer to activities that where the oxygen uptake is vigorously higher than the normal and he cardiac output is obtained. The activities include heavy weight lifting, aerobics exercises or regime, bicycling at high pace. Therefore, the data that is collected is based on the days/week hours and minutes/day.

Table 1.2. Distribution of responses according to activity

\begin{tabular}{|c|c|c|c|}
\hline Age Group & $\begin{array}{c}\text { Low } \\
\text { (Frequency) }\end{array}$ & $\begin{array}{c}\text { Moderate } \\
\text { (Frequency) }\end{array}$ & $\begin{array}{c}\text { High } \\
\text { (Frequency) }\end{array}$ \\
\hline $19-25$ & 44 & 49 & 28 \\
\hline $26-31$ & 11 & 6 & 1 \\
\hline $32-38$ & 0 & 1 & 0 \\
\hline $38-44$ & 5 & 0 & 0 \\
\hline $45-51$ & 3 & 3 & 0 \\
\hline
\end{tabular}

The low activity is slightly increased as compared to moderate activity .Therefore, the high/intense activity is decreased compared to both activity (moderate and low).

Mean \& Standard deviation of low, moderate \& high activity was $1547.24 \&$ standard deviation was 1728.37

Emotional Status: The emotional status is measured by the PERS questionnaire; the questionnaire is based on the scale that has several measurements which depend on one's response that are based on activation, intensity, and duration questionnaire that are proposed in two ways positive and negative.

The data is based on the negative (activation, intensity, duration) as well as on the positive (activation, intensity, duration).

- Negative activation: The negative activation is noted or determined when there are terms which are used like (upset, disappointed, frustrated, and negative.)

- Negative Intensity: The negative intensity is noted or determined when there are terms which are used like, upset, frustration, unhappy 
- Negative duration: the negative duration is noted or determined when there are terms which are used like, upset, anger, frustration, negative mood, and annoyed.

Distribution of negative reactivity: The mean value of negative reactivity was $2247 \&$ standard deviation for the negative reactivity was 113.859 (overall composite score) $150 \mathrm{n}$ numbers who had participated in the study

- Positive activation: The positive activation is noted or determined when there are terms which are used like, happy, positive, enthusiastic, good news

- Positive intensity: The positive intensity is noted or determined when there are terms which are used like, happiness, joyful, positive moods

- Positive duration: the positive duration is noted or determined when there are terms which are used like happy, feeling positive, enthusiastic, pleasant news, paying compliments.

Distribution of positive reactivity: The mean value of positive reactivity was 2187.33 and standard deviation of positive reactivity was 7.50 . $\mathrm{N}=150$ who had participated in the study

Count on for negativity vrs. positive reactivity: It shows that count on for negative verses positive reactivity shows that overall negative reactivity is higher than the positive reactivity.

\section{Result}

1. The low activity is slightly increased as compared to moderate activity.

2. The negative reactivity is higher than positive reactivity from the data that is collected.

3. The intense or moderate activity performed is less than the low activity or no activity

\section{Discussion}

The lockdown in COVID-19 is the pandemic effect of the 21 st century.

The effect of this pandemic outbreak is currently a point of discussion or a burning issue within the whole

Globalization, the scales which are used for determining the analysis for 'physical activity and emotional status' that proposes the certain answers. The vigorous physical activity which is proposed by WHO is quite $75 \mathrm{~min} /$ week $^{2}$. Therefore the data which is collected in this project determines that less than $30 \%$ of respondents have worked vigorously. The moderate physical activity which is proposed by WHO is quite

$150 \mathrm{~min} /$ week $^{2}$. The respondents that worked moderately physical activity are $42.8 \%$. As the physical inactivity can cause $17 \%$ lower risk to heart, 95\%confidence interval. Cardiovascular mortality can risk upto $23 \%$ and type 2 diabetes can be risked upto $26 \%$ according to WHO. There should be allowance of physical outdoor activities like running, walking or any other sports where there is an adequate amount of social distance is maintained where the pandemic covid-19 lockdown causes the consequences of cardiovascular diseases $^{2}$.

"With about five million dead, lack of exercise is the fourth most frequent cause of death globally in 2017"1. To check such data the 'no activity' analysis is a perfect response which determines that $80.3 \%$ of respondents have done no activity from the last 7 daysexcept sitting where the data intensifies 8 hours sitting .

The sector (college student and working sector) is the age of active stage, hence, the fact is that $78 \%$ of young men and $84 \%$ of young girls are affected by the lack of exercise ${ }^{1}$.As physical activity increases the immune system and reduces stress so the impact of physical activity is positive on each individual ${ }^{14}$. The consequences of homestay is no activity towards the healthy body are obesity, behavioral addictions disorders, exposure to sun is less (Vitamin D)and isolation from society ${ }^{10}$. The reductions in physical activity results in the emotional status which may be experienced such as negative or positive response according toevents ${ }^{2}$. Extended duration of indoor stay which cannot be avoided accompanied the binging time spent on watching television, playing games online through mobile and the break from socialization, that has worsen the behavioral (i.e. internet, screen, or television) addiction, disorders ${ }^{10}$. Longer the duration of lockdown increase in the PTSD symptoms ${ }^{14}$. The comparison of data with negative emotion with positive emotion. The scoring of negative emotions is well more than Positive emotions thought, physical activity affects the emotional reaction of an individual.

\section{Conclusion}

The resolving part is to check the physical activity 
and emotional status of college students and working sector people in a 1 month lockdown. The results that we found are negative with relation to physical activity and also negative with emotional status .As the article suggests the lack of activity difference one's mental or emotional ability.Though the data stand on this sentence. I would recommend that lack of physical activity not only affect the emotional status but also affect the cardiovascular activity,musculoskeletal activity, metabolic activity, hormonal activity. The research can further perform on old age and their physiology and psychology status. Within 1 month of span time of total lockdown in India. The data find out that there is a lot of variation in physical inactivity but not so much elevation disturbances in emotional status.

Ethical Clearance: It is the web survey study that is done in the institution (galgotias university) under the Department of Physiotherapy. This proposed project was placed before the Ethical Committee and has been approved as there is no objection to hold this project work.

Conflict of Interest: There is no conflict of interest of the author.

Funding of the Research: Self Funding.

\section{References:}

1. Pawlik L, Crises L. CAUSE OF DEATH: LACK OF MOVEMENT. https://www.academia.edu/ download/62494528/CAUSE_OF_DEATH_ LACK_OF_MOVEMENT_final.docx2020032662280-rjo9pe.pdf

2. Lippi G, Henry BM, Sanchis-Gomar F. Physical inactivity and cardiovascular disease at the time of coronavirus disease 2019 (COVID-19). European Journal of Preventive Cardiology. 2020 Apr 9:2047487320916823. https://journals.sagepub. com/doi/full/10.1177/2047487320916823

3. Brooks SK, Webster RK, Smith LE, Woodland L, Wessely S, Greenberg N, Rubin GJ. The psychological impact of quarantine and how to reduce it: rapid review of the evidence. The Lancet.2020,26Feb. https://www.sciencedirect. com/science/article/pii/S0140673620304608

4. Charansonney OL. Physical activity and aging: a life-long story. Discovery medicine. 2011 Sep9, 12(64):177-85. https://www.discoverymedicine. com/Olivier-L-Charansonney/2011/09/09/ physical-activity-and-aging-a-life-long-story/
5. Thompson PD, Franklin BA, Balady GJ, Blair SN, Corrado D, Estes III NM, Fulton JE, Gordon NF, Haskell WL, Link MS. Exercise and acute cardiovascular events:AHA Journal:2007; 27 April P 2358-68 https://www.ahajournals.org/doi/ full/10.1161/circulationaha.107.181485

6. Rhodes university ' $k$ keeping a lid on your mental health during lockdown " department of psychology,2020, Mon 30 Mach., https:// researchdata.ru.ac.za/articles/preprint/Keeping_a lid_on_your_mental_health_during_the lockdown/12258218

7. Rahiva R.Parveen 'inactivity during lockdown can impact bone health, says doctor.'The New Indian Express; Sunday, 2020 April 07th

8. Jenniser Boddy,Patrick O'Leary et.ll: 'Cabin Fever": the psychological impact of quarantine lockdown': Support independent journalism,2020 5 march.

9. ASP: 'Physical, mental health may suffer from being in Lockdown' relax news Free Malaysia today April 9, 2020 8:33 PM

10. Lippi G, Henry BM, Bovo C, Sanchis-Gomar F. Health risks and potential remedies during prolongedlockdowns for coronavirus disease; Oponion paper 2019 (COVID-19):26 March 2020, Diagnosis. 2020 Apr 7;1(ahead-of-print).

11. Hagströmer M, Oja P, Sjöström M. The International Physical Activity Questionnaire (IPAQ): a study of concurrent and construct validity. Public health nutrition. 2006 Sep;9(6):755-62.

12. Yoshi Iwasaka; Working with High Risk, Marginalized Youth: Youth-led Development of a Framework of Youth Engagement Youthrex research and evaluation eXchange 'international physical activity questionnaires' -short form revised august 2002.

13. Rodrigo Bacerra, David Preece Guillermo Campitelli,Glen scout -Pillow: Emotional reactivity-critical analysis and proposal of new scale: International journal of Applied psychology:2013;3(6),P 161-168

14. Frühauf A, Schnitzer M, Schobersberger W, Weiss $\mathrm{G}$, et al : Nordic walking and going for a walkinter-disciplinary recommendations to keep people physically active in times of the covid-19 lockdown in Tyrol,Austia. Current Issues in Sport Science (CISS). 2020 Apr 7.P 4-100 